\title{
Insulation Breakdown Influence Factors and Development of Cross-linked Polyethylene Cable
}

\author{
Yan-Li XU ${ }^{1, a}$, Wen-Jie MEI ${ }^{1, b,{ }^{*}}$, Hong-Liang YANG ${ }^{1, c}$, Chun-Mei $\mathrm{LI}^{1, \mathrm{~d}}$, \\ Guang-Hui SONG ${ }^{1, \mathrm{e}}$ \\ ${ }^{1}$ Jiangsu Hengtong High Voltage Power Cable System Co. Ltd, Suzhou, China \\ a249464811@qq.com, b892897377@qq.com, '82155556@qq.com, d605487189@qq.com, \\ e115947035@qq.com \\ ${ }^{*}$ Corresponding author
}

Keyword: XLPE, Space Charge, Water Tree, Mechanism.

\begin{abstract}
This paper mainly introduces the two cross-linking polyethylene insulation breakdown failure factors: space charge and water tree. And it further analyzes their mechanisms of initiation and growth. Cross-linking byproducts and current type are the major influence factors of space charge which will result in serious electric field distortion in the sample and to cause breakdown of insulation. Water tree is mainly influenced by cross-linking technology, impurities and temperature. Finally, this paper summarizes several influence factors and puts forward some improvement methods such as improving the crystallinity of materials, refining the spherulite size, homogenizing crystal, reducing the defects of cross-linked polyethylene.
\end{abstract}

\section{Introduction}

XLPE is widely used as the insulation of power cable currently because of the superior electrical, mechanical property and thermal stability ${ }^{[1]}$. With the increase of electrical load and rapid development of the island, rail transmit and industrialization, the demands of the HV and EHV power cable are larger than ever before ${ }^{[2]}$. However, the insulation will break down because of the various destruction from both inside and outside of the cable on operation. Once the insulation breaks down, it will have a big impact on the cable operation. The insulation deterioration and breakdown are the main factors that make the HV power cable out of operation. So, this phenomenon is focused by the whole industry. The global scholars have done many researches on this problem and they found insulation breakdown is a complicated process caused by the co-effect of electric, heat and the environment ${ }^{[3]}$. This paper summarizes the two cross-linking polyethylene insulation breakdown failure factors: space charge and water tree. It further analyzes their mechanism of initiation and growth. At the end of this paper, some relevant measures are put forward to improve the cable service life.

\section{Influence of Space Charge on Insulation Breakdown}

Space charge is defined as the existing net positive or negative charge between the heterogeneous phases or at the interface between electrode and medium of insulator or partial semi-conductor area. During the production, decomposing of DCP (Dicumyl peroxide) will generate the cross-linking by-product, which brings large amount of space charge. The high temperature during the degassing process will accelerate the release of the by-product and reduce the heteropolar space charge ${ }^{[4]}$. During operation, large amount of heteropolar space charge will appear on the surface of samples of the unaged DC (direct current) cable. While the heteropolar space charge will transform to homopolar space charge on the surface of samples of the aged DC cable ${ }^{[5]}$. Homopolar space charges will accumulate in the vicinity of the samples of the unaged AC (Alternating current) cable, but the amount is apparently smaller than the DC cable. Heteropolar space charge will accumulate in the vicinity of the samples of the aged AC cable. The space charge will distort the inner electric 
field and break down the insulation more easily.

\section{Formation Mechanism of Space Charge}

The cross-linked by-products can dissociate the polar groups and generate the heterocharges. The higher the electric field is, the more the heterocharges will appear. And these heterocharges will form the Space charge. The chain segments and end groups of the cross-linked by-product will form large amounts of "traps"[6]. There are such "traps" in the insulation as well. The "traps" consist of chemical flaws and structure flaws. Actually, these traps are caused by fractured chemical bond and small branch chain and most of them exist in the amorphous regions ${ }^{[7]}$. While the metal conductor is injecting the insulation with charge carriers, these traps will capture the charge carriers and form the space charge ${ }^{[8]}$. With the increase of the traps energy level, it is more difficult for the captured space charge to escape. As a result, large amounts of space charges accumulate and this area will be polarized seriously. Some charges will deviate from the traps once they are interfered by the outside environment which will lead to the depolarization of the medium. In this process, large amounts of energy will be released like the "avalanche" and finally the electric field is distorted ${ }^{[7]}$. However, the cross-linked by-product will reduce remarkably after the degassing. Thus the decrease of fractured chains and end groups will also reduce the traps, which will further improve the migration rate of sample charge carriers and make it easy for the electrons and holes to get away from the traps and reduce the space chares in the end ${ }^{[9]}$.

\section{Influence Factors of Water Tree}

Water tree aging is the main factor that will lead to the breakdown of the power cable when operating in the humid environment ${ }^{[10]}$. This phenomenon is initiated by penetration, saturation and condensation of water in the partial high electric field regions caused by impurity flaws, microvoids and semi-conductive protrusions in the insulation ${ }^{[11]}$. There are many factors that will affect the insulation water tree and the primary one is cross-linking technology. Mengkun Yan $^{[12]}$ did researches on the 5 different cross-linking technologies. He found the AC breakdown strength of inferior technology is $53.5 \%$ lower than the superior technology. The cross-linking process will have a significant effect on the crystalline forms which will then affect the growth of water tree. Xiaohui $\mathrm{Zhu}^{[13]}$ found three-dimensional structure will be formed during the cross-linking process. The degree of crystallinity is high and the distribution of crystal particles is homogeneous in suitable conditions, which will inhibit the formation of big crystals. During the degassing and cooling process, especially for the thick insulation, the outside of the insulation cools more quickly than the inside layer, which results in the formation of big and heterogeneous crystals on the inside of the insulation ${ }^{[14]}$. Jinfeng Wang ${ }^{[15]}$ did further researches on the chemical cross-linking technology and found the average growth speed of the water tree is quick for the few but big crystals. While the average growth speed of the water tree is slow for the many but small crystals.

The temperature will also affect the growth of the water tree. After the comparison of the experiments, it is found that amorphous state of XLPE with space charge will be oriented in the electric field when the temperature is smaller than the transition temperature (about $40^{\circ} \mathrm{C}$ ). Vertical to the oriented direction, the strength and modulus of materials are smaller than the one before oriented. It is easy to cause stress fatigue and fracture. And the water tree will grow quickly in this direction. When the temperature is higher than the transition temperature, the thermal motion of the molecule is in the leading position and the partial chain segments of the material are easily slacked. Under the Maxwell stress, it will cause stress fatigue and fracture and the water tree will grow quickly ${ }^{[16]}$. Above the $60^{\circ} \mathrm{C}$, in the macroscopic term, the higher the temperature is, the lower the property of materials will be. The microvoids will expand and the moisture and saline ions will accelerate diffusion, which make the water tree grow quickly ${ }^{[17]}$. On the other hand, aging can increase the TG activation energy to promote the cross-linking and optimize the physicochemical property of power cables when the temperature is below $100^{\circ} \mathrm{C}^{[18]}$.

\section{Initiation and Growth Mechanism of Water Tree}

The initiation and growth of water tree is a comprehensive and complex process under the impact 
of electric, chemical and mechanical actions. Jiankang Zhao ${ }^{[19]}$ did systematic researches and reduce to two theories: the mechanical destruction microcosmic mechanism and chemical reaction initiation mechanism. The mechanical destruction theory thinks as the following: after insulation absorbs water, high temperature heats the moisture inside the crack of the material, which will produce pressure. The alternating electric field impels the water bead inside the material to deform and produce the electrostriction force ${ }^{[17,20]}$ along the direction of electric field. When the outside pressure which is larger than the bond energy of the molecule chain is applied along the direction of electric field at the same time, the molecule chain will break. In these fractured areas, small holes will be formed and they will initiate the water tree. With the increase of these holes, the water tree will grow gradually. The chemical reaction mechanism thinks the water tree is just the product during the chemical reaction. Because oxygen can't be avoided within the cable, it will degrade the insulation at high temperature. The molecular chains will break and the numbers of carbonyl will increase $^{[21]}$. This degradation will form a gallery in amorphous area over time, which finally promotes the formation of water tree. However, the carbonyl or other strong polar groups will form a screen in the cusp of the water tree to inhibit the growth of water tree $e^{[22] .}$

In term of the induction and growth mechanism of water tree, we can design the longitudinal and radial water blocking structure for the cable to reduce the touch between water and insulation. Also, anti-oxygen, cross-linking agent and diene copolymer can be added to reduce the content of bound water inside the insulation. The normal operation temperature of XLPE cable is $90^{\circ} \mathrm{C}$, the microvoids, moisture and saline ions can diffuse quickly at this temperature. In the term of materials, improving the degree of crystallinity is useful because the crystalline region can prevent the diffusion and reduce the growth of water tree. In addition, improve the melting point can reduce the amorphous region caused by the rising of temperature.

\section{Research Prospects}

This paper analyzes the influences factors and initiation and growth mechanism. So we can draw the following conclusions from the analysis above.

a. No matter how to reduce the space charge, water tree or electric tree, we have to improve the degree of crystallinity, refine the spherocrystal and homogenize the crystal to reduce the flaws in XLPE. The specific methods need further researches.

b. The space charge can't be avoided during the cross-linking process. Nanoparticles can be added to the insulation materials as the donor holes and deep traps of the overlapped centre of both positive and negative charges. However it is harmful to the improvement of degree of crystallinity. At present, conductor pre-heating is a general method to reduce the crystal imperfection on the inner layer of insulation. Other methods are under consideration.

c. Other methods are taken during production such as blending the insulation and cross-linking agent well and controlling the reaction temperature and production speed reasonably to conduct the cross-linking reaction completely, cleaning the vulcanization pipeline periodically to reduce the accumulation of cross-linking by-product on the pipeline wall, low temperature and long time cooling during degassing which can reduce the microvoids on the inner layer, preventing the core from scratch and damage and so on. In consideration of the voltage rating of power cable is higher and higher, the thickness of insulation is increasing at the same time. The control of cross-linking is more and more difficult. So it is important to do further researches on the insulation.

\section{References}

[1] M.I.Qureshi; N.H.Malik.etc. A.A.Al-Arainy. Impact of cations toward the water tree propensity in crosslinked polyethylene insulation [J], Journal of King Saud University -Engineering Science, 2011, 18(5):43-48.

[2] Chengcheng Zhang; Chunyang Li; Hong Zhao, etc. A review on the aging performance of direct current cross-linked polyethylene insulation materials [J], Properties and Applications of Dielectric 
Materials , 19-22 July 2015, 700-703.

[3] Boxue Du; Zongle Ma; Yu Gao,etc; Insulation evaluation of water-tree aged 10kV XLPE cables using thermal step method [J], High voltage Engineering, Vol 37, No.1 January 31, 2011: 143-149. (in Chinese)

[4] Wendong Deng; Fanyu Yuan; Jun Li, etc; Thermal aging behavior of XLPE cable based on the nuclear magnetic resonance theory [J], Journal of Chongqing University, Vol 37, No.8 2014: 41-45. (in Chinese)

[5] Fuzeng Zhang; Shuqi Li; Yonghua Zhu, etc; Influence of Thermal Ageing on Space Charge Characteristics of Extruded XLPE HVDC Cable[J], Insulation Material Vol 48, No.8 2014: 41-45. (in Chinese)

[6] Qiongxia Zhong; Li Lan; Jiandong Wu, etc. The Influence of Cross-Linked By-products on Space Charge Behavior in XLPE [J], Proceedings of the CSEE, Vol 35, No.11 June 5 2015:2903-2910. (in Chinese)

[7] Shengtao Li; Qifeng Huang; Jian Sun, etc. Influence of Aggregation Structure and Traps on Surface Flashover of XLPE in Vacuum [J], Acta Physica Sinica, Vol 59, No.1 2010:422-428. (in Chinese)

[8] Junguo Gao; Hao Zhang; Lili Li, etc. Study on Space Charge Characteristics of XLPE/O-MMT Nano composites [J], Acta Polymerica Sinica, No.1 2013:126-133. (in Chinese)

[9] Deming Tu; Xia Wang; Zepeng Lv, etc. Formation and Inhibition Mechanisms of Space Charges in Direct Current XLPE Insulation Explained by Energy Band Theory [J], Acta Physica Sinica, Vol 61, No.1 2012: 017104-1-017104-7. (in Chinese)

[10] Chonung Kim, Jingkuan Duan, Xingyi Huang. etc. Numerical analysis on water treeing deterioration of XLPE cable insulation using combination of FEM and Taguchi method [J], Electrical energy systems,20(6):747-759.

[11] Xiangrong Chen; Yang Xu; Ying Liu, etc. Study on Conducting Characteristics of Electrical Tree in XLPE Cable Insulation [J], Acta Physica Sinica, Vol 61, No.8 2012: 087701 :1-9. (in Chinese)

[12] Mengkun Yan; Mingzhong Xu; Fugui Miao, etc. Effects of Various Vulcanization Methods on Power Frequency Breakdown Property of Water Treeing Retardant XLPE Power Cable [J], High Voltage Engineering, Vol 38, No.9 September 30 2012: 2424-2429. (in Chinese)

[13] Xiaohui Zhu; Boxue Du; Yu Gao, etc. Effects of Crossing Linking Process on Crystallinity of XLPE [J], Insulating Materials, Vol 43, No.6 2010: 44-47. (in Chinese)

[14] Yitian Wang; Xiaoquan Zheng; G Chen; A E Davies etc; Influence of Polymer Congregating State and Survival Mechanical Stress to Electrical Treeing in XLPE [J], Transactions of China Electrotechnical Society, Vol 19, No.7 2004: 44-48. (in Chinese)

[15] Jinfeng Wang; Zhiming Liu; Yanxiong Li, etc; Influence of Chemical Cross-linking on Water Treeing in Polyethylene [J], High Voltage Engineering, Vol 37, No.10 2011: 2477-2484. (in Chinese)

[16] Kai Zhou; Wenbiao Tao; Wei Zhao, etc. Towards Understanding the Characteristics of Water Tree Growth at Different Temperatures in XLPE Based on the Theory of Molecular Orientation [J], High Voltage Engineering, Vol 40, No.12 2014: 3665-3673. (in Chinese)

[17] Jinfeng Wang; Zhiming Liu; Yanxiong Li, etc; Influence of Temperature on Water Treeing in Polyethylene [J], High Voltage Engineering, Vol 38, No.1 2012: 181-187. (in Chinese) 
[18] Jun Xu; Xiaodong Wang; Benhong Ouyang etc; Effect of Thermal Aging on the Physicochemical Structure of XLPE Cable Insulation [J], Insulation Materials , Vol 46, No.2 2013: 33-37. (in Chinese)

[19] Jiankang Zhao; Benhong Ouyang; Xuetong Zhao etc; Review of Influence of Water-tree on Microstructure and Properties of XLPE Cable Insulation Materia 1[J], Insulation Materials, Vol 43, No.5 2010: 50-55. (in Chinese)

[20] Chengchenng Zhang; Chunyang Li; Ziang Jiang;etc. Research on the water blade electrode method for assessing water tree resistance of cross-linked polyethylene [J], Polymer Testing, Volume 50, April 2016, Pages 235-240.

[21] Hang Wang; Guoxin Tan; Ying Tan, etc; Life Span and Physicochemical Property Analysis of XLPE submarine cable insulation [J], Polymer Materials Science \& Engineering, Vol 31, No.3 2015: 71-75. (in Chinese)

[22] Tianxiong Jin; Xingyi Huang; Kaiping Jiang etc; Investigation on Influence of Thermal Aging on Water Tree Inception in XLPE Cable Insulation[J], Insulating Materials, Vol 40, No.5 2007: 45-49. (in Chinese) 